\title{
Finite-Time Chaotic Control of Unified Hyperchaotic Systems with Multiple Parameters
}

\author{
Chang-Zhong Chen ${ }^{1,3}$, Ping $\mathrm{He}^{2}$, Tao Fan ${ }^{1,3}$ and Chun-Guo Jing ${ }^{4}$ \\ 1 School of Automation and Electronic Information, Sichuan University of Science \\ \& Engineering, Zigong, Sichuan 643000, People's Republic of China \\ c-zchen@qq.com \\ 2 Department of Electromechanical Engineering, Faculty of Science and \\ Technology, E11, University of Macau, Avenida da Universidade, Taipa, 999078, \\ Macao Special Administrative Region of the People's Republic of China. \\ pinghecn@126.com; pinghecn@qq.com \\ 3 Artificial Intelligence Key Laboratory of Sichuan Province, Sichuan University \\ of Science \& Engineering, Zigong, Sichuan 643000, People's Republic of China. \\ fantaoid@qq.com \\ 4 School of Computer and Communication Engineering, Northeastern University \\ at Qinhuangdao, Qinhuangdao, Hebei, 066004, People's Republic of China. \\ jingchunguo@163.com
}

\begin{abstract}
This paper is concerned with finite-time chaos control of unified hyperchaotic systems with multiple parameters. Based on the finite-time stability theory in the cascade-connected systems, a nonlinear control law is presented to achieve finite-time chaos control. The controller is simple and easy to be constructed. Simulation results for Lorenz hyperchaotic system, $L \ddot{u}$ hyperchaotic system, Chen hyperchaotic system are provided to illustrate the effectiveness of the proposed scheme.
\end{abstract}

Keywords: Finite-time chaos control, Unified hyperchaotic system, Cascade-connected system

\section{Introduction}

Chaotic system has attracted much attention because of its powerful application in the areas of secure communication, information processing, biological engineering, and chemical processing [1-9]. As a new subject in 1980s, chaos almost covers all the fields of science. It is known that chaos is an interesting nonlinear phenomenon which may lead to irregularity and unpredictability in the dynamic system, and it has been intensively studied in the last three decades. Since Pecora and Carroll proposed the PC method to synchronize two chaotic systems in $1990[10,11]$, the study of synchronization of chaotic systems has been widely investigated due to their potential applications in various fields, for instance, chemical reactions, biologicals systems, and secure communication. Over the past decades, a variety of control approaches such as adaptive control [12], linear feedback control [13], active control [14], and back stepping control [15] have been proposed for various types of synchronization, which include complete synchronization [16], projective synchronization [17-18], general synchronization [19], lag synchronization [20], and novel compound synchronization [21].

Notice that the mentioned literatures mainly investigated the asymptotic synchronization of chaotic systems. However, in the view of practical application, optimizing the synchronization time is more important than achieving synchronization asymptotically [22-26]. Recently, based on the step-by-step control method, Wang et al. 
realized the finite-time synchronization of two chaotic systems by designing a proper controller [22]. The method has the ability to achieve global stability in finite time. In addition, the step-by-step technique has the advantage of reducing controller complexity.

In this paper, we deal with the so-called unified hyperchaotic systems presented in [27], which is a generalized form of the hyperchaotic L"u system, hyperchaotic Chen system, and hyperchaotic Lorenz system. Based on the finite-time stability theory and stability theory of cascade-connected system, we present a new continuous controller to realize finite-time chaos control for the unified hyperchaotic systems.

\section{Preliminary Definitions and Lemmas}

Finite-time stability means that the state of the dynamic system converges to a desired target (in general referring to the origin) within a finite time. First, let us introduce some necessary definitions and lemmas.

Lemma 1 (see [22]) For the system,

$$
\dot{x}=f(x) \text {. }
$$

Assume that a continuous, positive-definite function $V(t)$ satisfies the following differential inequality:

$$
\dot{V}(t) \leq-c V^{\eta}(t), \quad \forall t \geq t_{0}, \quad V\left(t_{0}\right) \geq 0,
$$

where $c>0$ and $0<\eta<1$ are constants. Then, for any initial time $t_{0}, V(t)$ satisfies

$$
\begin{gathered}
V^{1-\eta}(t) \leq V^{1-\eta}\left(t_{0}\right)-c(1-\eta)\left(t-t_{0}\right), \quad t_{0} \leq t \leq t_{1}, \\
V(t) \equiv 0, \quad \forall t \geq t_{1},
\end{gathered}
$$

with $t_{1}$ given by

$$
t_{1}=t_{0}+\frac{V^{1-\eta}\left(t_{0}\right)}{c(1-\eta)}
$$

Thus, for any initial value $V\left(t_{0}\right)$, the system (1) has $V(t)=0$ in $t_{1}=t_{0}+V^{1-\eta}\left(t_{0}\right) / c(1-\eta)$, that is, the system can achieve global stability in finite time.

Lemma 2 Let $0<c<1$. Then for positive real numbers $a$ and $b$, the following inequality holds

$$
(a+b)^{c} \leq a^{c}+b^{c} .
$$

This result is quite straight forward and the proof is omitted here.

\subsection{Main Results}

It is known that a chaotic system has sensitive dependence on its initial conditions and parameters. Before 1990, most researchers thought that the chaotic systems cannot be controlled to a desired target. However, in 1990, the pioneering work of Ott, Grebogi, and Yorke [28] denied completely the viewpoint. In this section, our aim is to design a control law that realizes finite-time chaos control for the unified hyperchaotic systems. We start with considering the finite-time stable problem for the case of certain parameters. Then we turn the problem to the system with uncertain parameters.

The unified hyperchaotic system can be described as 


$$
\left\{\begin{array}{l}
\dot{x}_{1}=(25 a+20)\left(x_{2}-x_{1}\right)+x_{4}, \\
\dot{x}_{2}=(28-35 a) x_{1}+(29 a-1) x_{2}-x_{1} x_{3}, \\
\dot{x}_{3}=-\frac{8+a}{3} x_{3}+x_{1} x_{2} \\
\dot{x}_{4}=b x_{4}+c x_{2} x_{3}
\end{array}\right.
$$

where $\left[x_{1}, x_{2}, x_{3}, x_{4}\right]^{T}$ is the state variables group of the unified hyperchaotic system, and $a \in[0,1]$ is a parameter [27].

Because the system (2) is belong to the generalized Lorenz hyperchaotic system for $\mathrm{a}=$ $0, \mathrm{~b}=-1, \mathrm{c}=-1$, it belongs to Lü hyperchaotic system for $\mathrm{a}=0.8, \mathrm{~b}=0.3, \mathrm{c}=0.1$ and belongs to generalized Chen hyperchaotic system for $\mathrm{a}=1, \mathrm{~b}=0.2, \mathrm{c}=0.1$, so we consider the system (2) as unified hyperchaotic system. The hyperchaotic attractor are shown in Figure1-3, respectively.
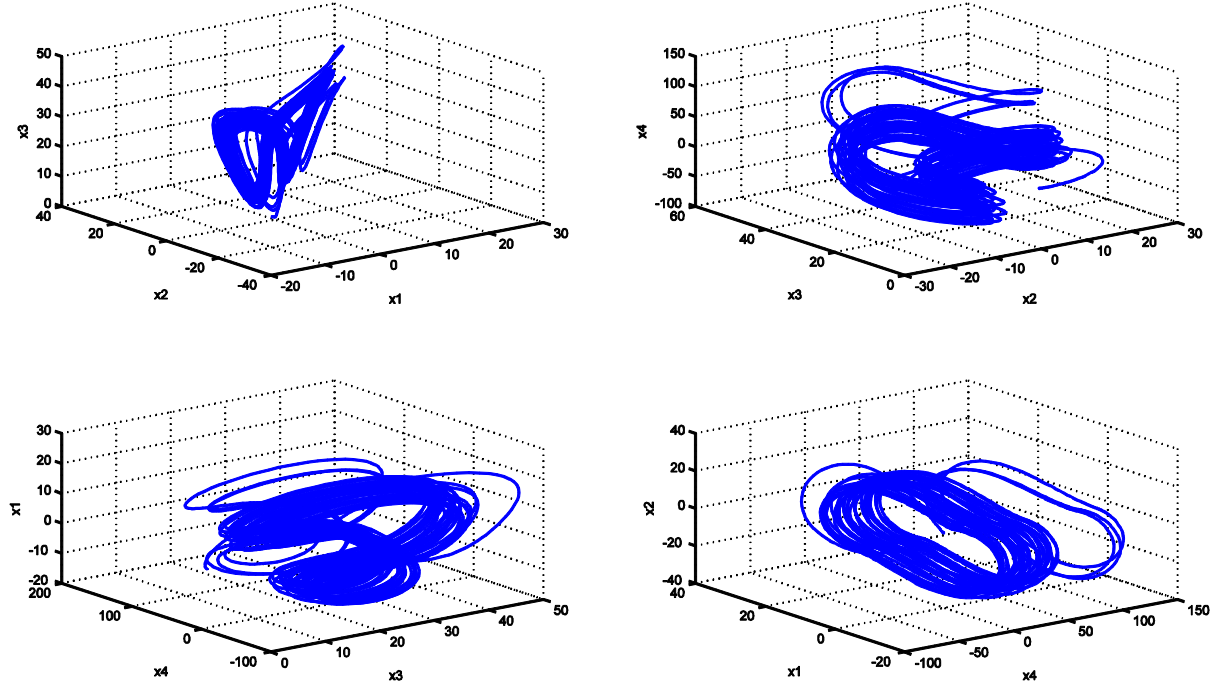

Figure 1. Phase Planes of Lorenz Hyperchaotic System
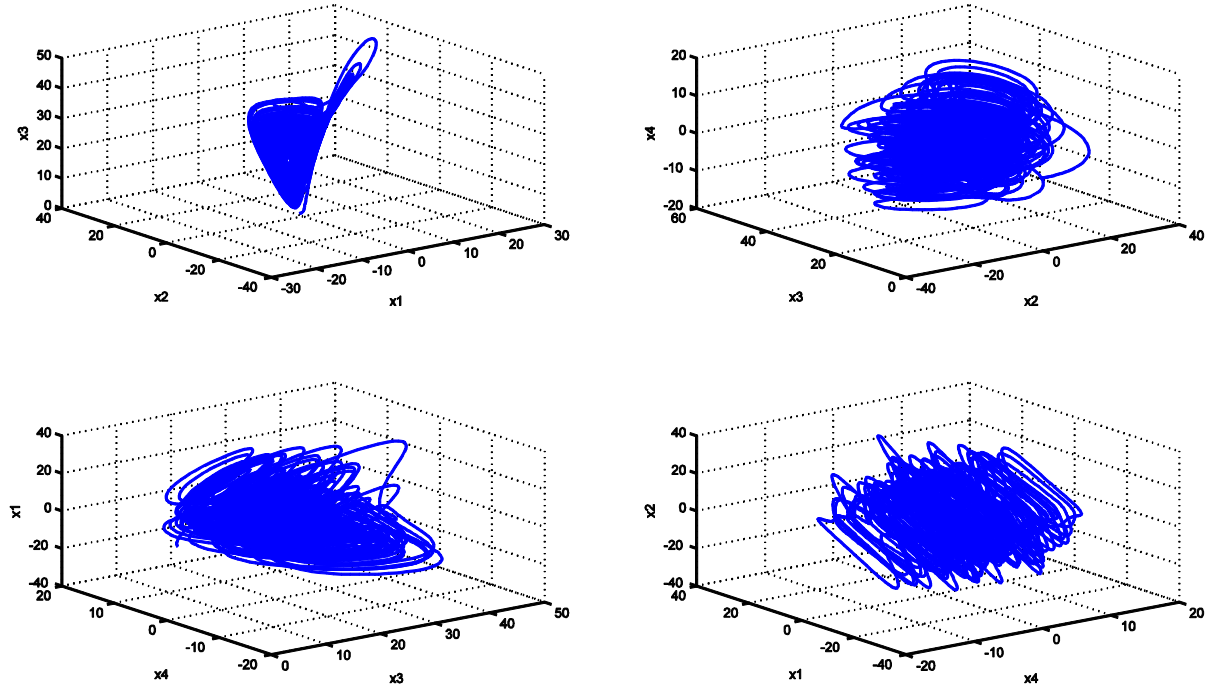

Figure 2. Phase Planes of Lü Hyperchaotic System 

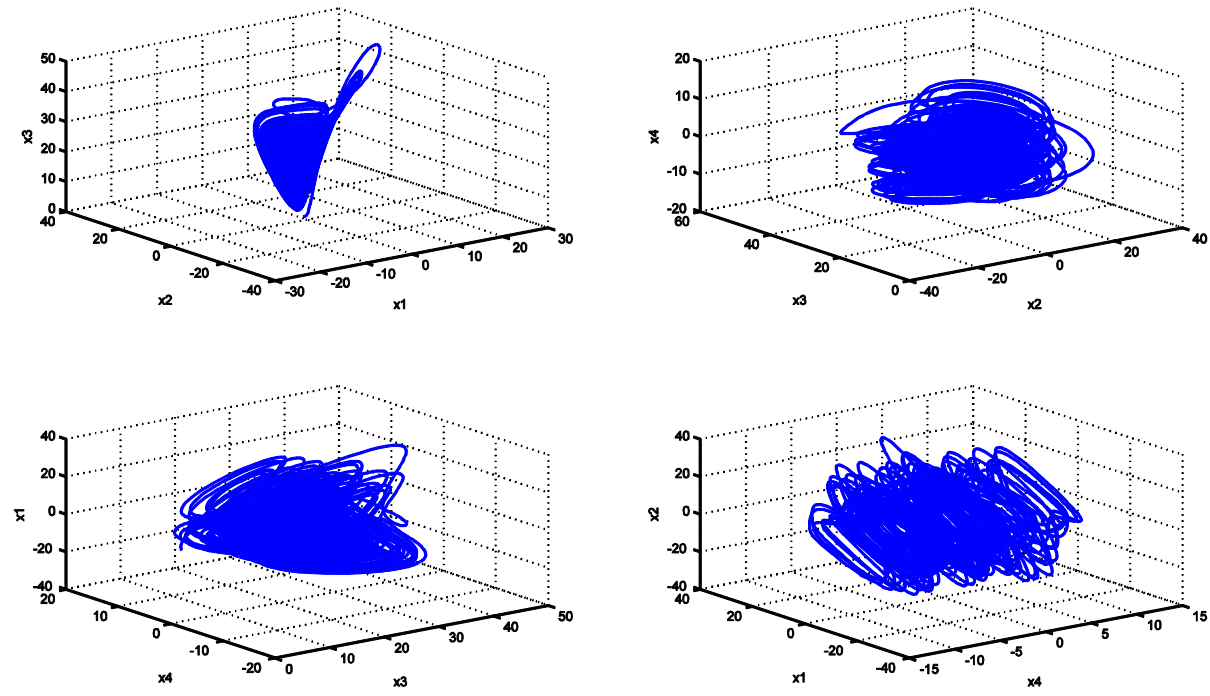

Figure 3. Phase Planes of Chen Hyperchaotic System

In this section, we consider the unified chaotic system with certain parameters, and design a controller to globally stabilize the unstable equilibrium $\mathrm{o}=[0,0,0,0]^{\mathrm{T}}$ in a finite time. As to other equilibria, we can also adopt the technique presented below to realize the chaos control.

The controlled unified chaotic systems can be described by

$$
\left\{\begin{array}{l}
\dot{x}_{1}=(25 a+20)\left(x_{2}-x_{1}\right)+x_{4}+u_{1}, \\
\dot{x}_{2}=(28-35 a) x_{1}+(29 a-1) x_{2}-x_{1} x_{3}+u_{2}, \\
\dot{x}_{3}=-\frac{8+a}{3} x_{3}+x_{1} x_{2}+u_{3}, \\
\dot{x}_{4}=b x_{4}+c x_{2} x_{3}+u_{4}
\end{array}\right.
$$

where $u_{1}, u_{2}, u_{3}$ and $u_{4}$ are controllers. The design procedure is divided into three steps.

Step 1 Let $u_{1}=-(25 a+20) x_{2}-x_{4}-x_{1}^{\beta}$, and where are positive odd integers. By the controller, the first equation of (3) is

$$
\dot{x}_{1}=-(25 a+20) x_{1}-x_{1}^{\beta} \text {. }
$$

Consider the candidate Lyapunov function $V_{1}=\frac{1}{2} x_{1}^{2}$. The time derivative of $V_{1}$ along the trajectory of (4) is 


$$
\begin{aligned}
\dot{V}_{1} & =x_{1}\left(-(25 a+20) x_{1}-x_{1}^{\beta}\right) \\
& =-(25 a+20) x_{1}^{2}-x_{1}^{\beta+1} \\
& \leq-x_{1}^{\beta+1} \\
& =-\left(\frac{1}{2}\right)^{-\frac{\beta+1}{2}}\left(\frac{1}{2} x_{1}^{2}\right)^{\frac{\beta+1}{2}} \\
& =-\left(\frac{1}{2}\right)^{-\frac{\beta+1}{2}}\left(V_{1}\right)^{\frac{\beta+1}{2}} .
\end{aligned}
$$

Since $0<\beta<1$, then $0<\frac{\beta+1}{2}<1$. From Lemma 1 , the state variable $x_{1}$ will reach $x_{1}=0$ at a finite time $T_{1}=\frac{x_{1}(0)}{1-\beta}$.

Step 2 Let $u_{2}=-L_{1} x_{2}-x_{2}^{\beta}$, where $L_{1} \geq 29 a-1$, and $u_{3}=-x_{3}^{\beta}$. If $t>T_{1}$, then $x_{1} \equiv 0$.

Substituting $x_{1}=0$ into the second and the third equations of system (3), it yields

$$
\left\{\begin{array}{l}
\dot{x}_{2}=(29 a-1) x_{2}-L_{1} x_{2}-x_{2}^{\beta}, \\
\dot{x}_{3}=-\frac{8+a}{3} x_{3}-x_{3}^{\beta} .
\end{array}\right.
$$

Choose Lyapunov function for (6) as follows

$$
V_{2}=\frac{1}{2} x_{2}^{2}+\frac{1}{2} x_{3}^{2}
$$

The derivative of $V_{2}$ along the trajectories of (6) is

$$
\begin{aligned}
\dot{V}_{2} & =x_{2}\left((29 a-1) x_{2}-L_{1} x_{2}-x_{2}^{\beta}\right)-x_{3}\left(\frac{8+a}{3} x_{3}+x_{3}^{\beta}\right) \\
& =-\left(L_{1}-(29 a-1)\right) x_{2}^{2}-x_{2}^{\beta+1}-\frac{8+a}{3} x_{3}^{2}-x_{3}^{\beta+1} \\
& \leq-x_{2}^{\beta+1}-x_{3}^{\beta+1} \\
& =-\left(\frac{1}{2}\right)^{-\frac{\beta+1}{2}}\left(\frac{1}{2} x_{2}^{2}\right)^{\frac{\beta+1}{2}}-\left(\frac{1}{2}\right)^{-\frac{\beta+1}{2}}\left(\frac{1}{2} x_{3}^{2}\right)^{\frac{\beta+1}{2}} \\
& =-\left(\frac{1}{2}\right)^{-\frac{\beta+1}{2}}\left(\left(\frac{1}{2} x_{2}^{2}\right)^{\frac{\beta+1}{2}}+\left(\frac{1}{2} x_{3}^{2}\right)^{\frac{\beta+1}{2}}\right) \\
& \leq-\left(\frac{1}{2}\right)^{-\frac{\beta+1}{2}}\left(\frac{1}{2} x_{2}^{2}+\frac{1}{2} x_{3}^{2}\right)^{\frac{\beta+1}{2}} \\
& =-\left(\frac{1}{2}\right)^{-\frac{\beta+1}{2}} V_{2}^{\frac{\beta+1}{2}} .
\end{aligned}
$$


It should be pointed out that we have used Lemma 2 to deduce the above result. Since $0<\beta<1,0<\frac{\beta+1}{2}<1$. Form Lemma 1, the state variables $x_{2}$ and $x_{3}$ will converge to $x_{2}=0, x_{3}=0$ at a finite time $T_{2}=T_{1}+\frac{x_{3} T_{1}}{1-\beta}$.

Step 3 Let $u_{4}=-L_{2} x_{4}-x_{4}^{\beta}$, where $L_{2} \geq b$. If $t>T_{1}+T_{2}$, then $x_{1} \equiv x_{2} \equiv x_{3}=0$. Substituting $x_{2}=0$ into the fourth equations of system (3), it yields

$$
\dot{x}_{4}=-\left(L_{2}-b\right) x_{4}-x_{4}^{\beta} .
$$

Consider the Lyapunov function

$$
V_{3}=\frac{1}{2} x_{4}^{2}
$$

The time derivative of $\mathrm{V} 3$ along the trajectories of (9) is

$$
\begin{aligned}
\dot{V}_{3} & =x_{4}\left(-\left(L_{2}-b\right) x_{4}-x_{4}^{\beta}\right) \\
& =-\left(L_{2}-b\right) x_{4}^{2}-x_{4}^{\beta+1} \\
& \leq-x_{4}^{\beta+1} \\
& =-\left(\frac{1}{2}\right)^{-\frac{\beta+1}{2}}\left(\frac{1}{2} x_{4}^{2}\right)^{\frac{\beta+1}{2}} \\
& =-\left(\frac{1}{2}\right)^{-\frac{\beta+1}{2}}\left(V_{3}\right)^{\frac{\beta+1}{2}} .
\end{aligned}
$$

Since $0<\beta<1$, then $0<\frac{\beta+1}{2}<1$. Form Lemma 1 , the state variable $x_{4}$ will reach $x_{4}=0$ at a finite time $T_{3}=T_{2}+\frac{x_{4} T_{2}}{1-\beta}$.

Then after $T_{3}$, the state of the system (3) will stay at $x_{1}=0, x_{2}=0, x_{3}=0$, $x_{4}=0$. This means the controlled unified hyperchaotic systems (3) are finite-timely stabilized by the controller

$$
\left\{\begin{array}{l}
u_{1}=-(25 a+20) x_{2}-x_{4}-x_{1}^{\beta}, \\
u_{2}=L_{1} x_{2}-x_{2}^{\beta}, \\
u_{3}=-x_{3}^{\beta}, \\
u_{4}=-L_{2} x_{4}-x_{4}^{\beta},
\end{array}\right.
$$

where $L_{1} \geq 29 a-1, \quad L_{2} \geq b$.

\subsection{Simulation Results}

In this section, to demonstrate the effectiveness of the proposed method, we present the simulation results for the unified hyperchaotic systems. In the numerical simulations, the fourth-order Runge-Kutta method is used to solve the systems with time step size 0.001. The initial condition of the chaotic system is always adopted as 
$\left[x_{1}(0), x_{2}(0), x_{3}(0), x_{4}(0)\right]^{T}=[-0.5,0.5,1,-1]^{T}$. Figures (4)-(6) show the simulation results for the Lorenz, Lü and Chen hyperchaotic systems, respectively. The controller gains are chosen as $L_{1}=29 a+1, \beta=\frac{2}{3}$. From the figures, we can see the state reaches the origin in a finite time.
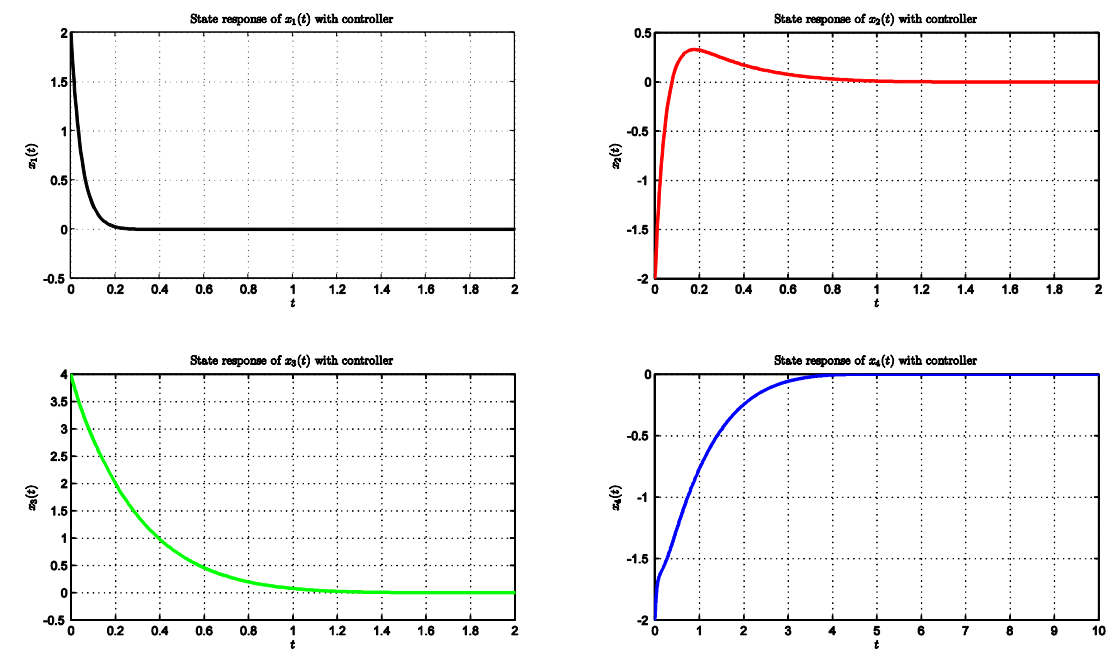

Figure 4. Time Response of Lorenz Hyperchaotic System
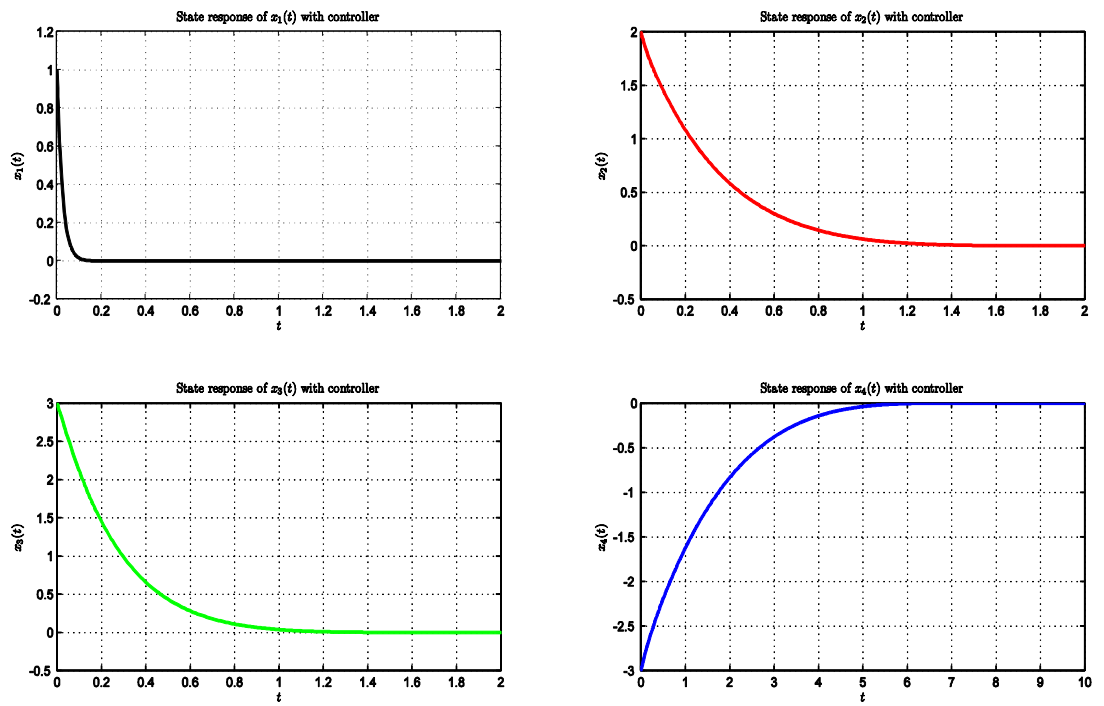

Figure 5. Time Response of Lü Hyperchaotic System 

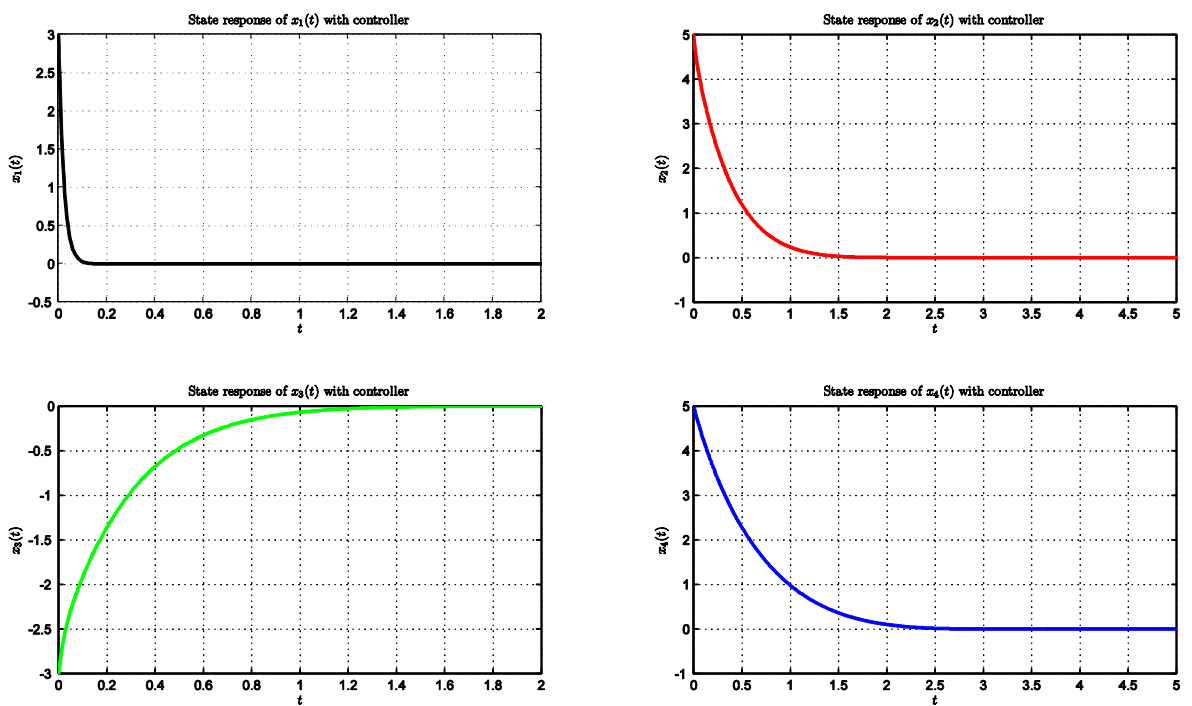

Figure 6. Time Response of Chen Hyperchaotic System

\section{Conclusion}

In this paper, the problem of finite-time chaos control for the unified hyperchaotic systems was investigated. Based on the finite-time stability theory, the step-by-step control and nonlinear control approach, a suitable controller was introduced. The simulation results demonstrated that the proposed controller works well for synchronizing three hyperchaotic systems in finite dime. From the proofs, we can see that this method can be extended to other chaotic systems.

\section{Acknowledgments}

This work was jointly supported by the Breeding Project Foundation of Sichuan University of Science and Engineering (Grant No. 2014PY14), the Research Foundation of Department of Education of Sichuan Province (Grant Nos. 14ZA0203 and 14ZB0210), the Open Foundation of Enterprise Informatization and Internet of Things Key Laboratory of Sichuan Province (Grant Nos. 2014WYJ01 and 2013WYY06), the Open Foundation of Artificial Intelligence Key Laboratory of Sichuan Province (Grant Nos. 2014RYY02, 2013RYJ01, and 2012RYJ01), the National Natural Science Foundation of China (Grant Nos. 61203001 and 61473066), the Program for New Century Excellent Talents in University (No. NCET-12-0103), and the Science Foundation of Sichuan University of Science and Engineering (Grant No. 2012KY19).

\section{References}

[1] P. Colet and R. Roy, "Digital communication with synchronized chaotic lasers", Optics Letters, vol. 19, no. 24, (1994), pp. 2056-2058.

[2] J. Lu, X. Wu, and J. Lü, "Synchronization of a unified chaotic system and the application in secure communication", Physics Letters A, vol. 305, no. 6, (2002), pp. 365-370.

[3] C.-G. Jing, P. He, T. Fan, Y. Li, and X. Chen, Changzhong ang Song, "Single state feedback stabilization of unified chaotic systems and circuit implementation", Open Physics, vol. 13, no. 1, (2015), pp. 111-122.

[4] P. He, C.-G. Jing, T. Fan, and C.-Z. Chen, "Robust decentralized adaptive synchronization of general complex networks with coupling delayed and uncertainties", Complexity, vol. 19, no. 3, (2014), pp. 10-26. 
[5] P. He, Q. Zhang, C.-G. Jing, C.-Z. Chen and T. Fan, "Robust exponential synchronization for neutral complex networks with discrete and distributed time-varying delays: A descriptor model transformation method", Optimal Control Applications and Methods, vol. 35, no. 6, (2014), pp. 676-695.

[6] P. He, C.-G. Jing, C.-Z. Chen, T. Fan, and H. S. Nik, "Synchronization of general complex networks via adaptive control schemes", Pramana - journal of physics, vol. 82, no. 3, (2014), pp. 499-514.

[7] P. He, C.-G. Jing, T. Fan, and C.-Z. Chen, "Outer synchronization of complex networks with multiple coupling time-varying delays", International Journal of Control \& Automation, vol. 6, no. 4, 2013, pp. $13-32$.

[8] P. He, C.-G. Jing, T. Fan, and C.-Z. Chen, "Robust adaptive synchronization of complex networks with multiple coupling time-varying delays", International Journal of Automation and Control, vol. 7, no. 4, (2013), pp. 223-248.

[9] C.-Z. Chen, T. Fan, B.-R. Wang, H. S. Nik, and P. He, "Feedback linearization synchronization of unified chaotic systems", Journal of Applied Nonlinear Dynamics, vol. 3, no. 2, (2014), pp. 173-186.

[10] L. M. Pecora and T. L. Carroll, "Synchronization in chaotic systems", Physical Review Letters, vol. 64, no. $8,(\mathbf{1 9 9 0})$, pp. $821-824$

[11] L. M. Pecora, T. L. Carroll, G. A. Johnson, D. J. Mar, and J. F. Heagy, "Fundamentals of synchronization in chaotic systems, concepts, and applications", Chaos: An Interdisciplinary Journal of Nonlinear Science, vol. 7, no. 4, (1997), pp. 520-543.

[12] T.-L. Liao and S.-H. Tsai, "Adaptive synchronization of chaotic systems and its application to secure communications", Chaos, Solitons \& Fractals, vol. 11, no. 9, (2000), pp. 1387-1396.

[13] M. T. Yassen, "Controlling chaos and synchronization for new chaotic system using linear feedback control", Chaos, Solitons \& Fractals, vol. 26, no. 3, (2005), pp. 913-920.

[14] Y. Lei, W. Xu, and H. Zheng, "Synchronization of two chaotic nonlinear gyros using active control", Physics Letters A, vol. 343, no. 1, (2005), pp. 153-158.

[15] S. Tong, C. Li, and Y. Li, "Fuzzy adaptive observer backstepping control for mimo nonlinear systems", Fuzzy Sets and Systems, vol. 160, no. 19, (2009), pp. 2755-2775.

[16] C. Yao, Q. Zhao, and J. Yu, "Complete synchronization induced by disorder in coupled chaotic lattices", Physics Letters A, vol. 377, no. 5, (2013), pp. 370-377.

[17] S. Jun-Wei, S. Yi, Z. Guo-Dong, W. Yan-Feng, and C. Guang-Zhao, "General hybrid projective complete dislocated synchronization with non-derivative and derivative coupling based on parameter identification in several chaotic and yperchaotic systems", Chinese Physics B, vol. 22, no. 4, (2013), 040508.

[18] J. Sun, Y. Shen, and G. Zhang, "Transmission projective synchronization of multisystems with non-delayed and delayed coupling via impulsive control", Chaos: An Interdisciplinary Journal of Nonlinear Science, vol. 22, no. 4, (2012), pp. 043107.

[19] H. G. Enjieu Kadji and R. Yamapi, "General synchronization dynamics of coupled van der pol-duffing oscillators", Physica A: Statistical Mechanics and its Applications, vol. 370, no. 2, (2006), pp. 316-328.

[20] Y. Shen and J. Wang, "Robustness analysis of global exponential stability of recurrent neural networks in the presence of time delays and random disturbances", IEEE Transactions on Neural Networks and Learning Systems, vol. 23, no. 1, (2012), pp. 87-96.

[21] J. Sun, Y. Shen, Q. Yin, and C. Xu, "Compound synchronization of four memristor chaotic oscillator systems and secure communication", Chaos: An Interdisciplinary Journal of Nonlinear Science, vol. 23, no. 1, (2013), 013140.

[22] H. Wang, Z.-z. Han, Q.-y. Xie, and W. Zhang, "Finite-time chaos synchronization of unified chaotic system with uncertain parameters", Communications in Nonlinear Science and Numerical Simulation, vol. 14 , no. 5, (2009), pp. 2239-2247.

[23] Y. Yang and $\mathrm{X}$. Wu, "Global finite-time synchronization of a class of the nonautonomous chaotic systems", Nonlinear Dynamics, vol. 70, no. 1, (2012), pp. 197-208.

[24] U. E. Vincent and R. Guo, "Finite-time synchronization for a class of chaotic and hyperchaotic systems via adaptive feedback controller", Physics Letters A, vol. 375, no. 24, (2011), pp. 2322-2326.

[25] L. Runzi and W. Yinglan, "Finite-time stochastic combination synchronization of three different chaotic systems and its application in secure communication", Chaos: An Interdisciplinary Journal of Nonlinear Science, vol. 22, no. 2, (2012), pp. 023109.

[26] P. He, S.-H. Ma, and T. Fan, "Finite-time mixed outer synchronization of complex networks with coupling time-varying delay", Chaos: An Interdisciplinary Journal of Nonlinear Science, vol. 22, no. 4, (2012), pp. 043151.

[27] X.-Y. Wang and G.-B. Zhao, "Hyperchaos generated from the unified chaotic system and its control", International Journal of Modern Physics B, vol. 24, no. 23, (2010), pp. 4619-4637.

[28] E. Ott, C. Grebogi, and J. A. Yorke, "Controlling chaos", Physical Review Letters, vol. 64, no. 11, (1990), pp. 1196-1199. 


\section{Authors}

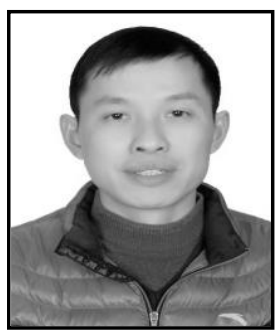

Chang-Zhong Chen received the B.S. Degree in Automatic Control from Sichuan University, in Chengdu, Sichuan, People's Republic of China and the M.S. Degrees in Control Science and Engineering from Sichuan University of Science \& Engineering, in Zigong, Sichuan, China, in 1997 and 2009, respectively. He is currently an Associate Professor with the School of Automation and Electronic Information, Sichuan University of Science \& Engineering, in Zigong, Sichuan, China, from 2010. His research interests include Technology and application of microcontrollers, Intelligent control and Chaos control.

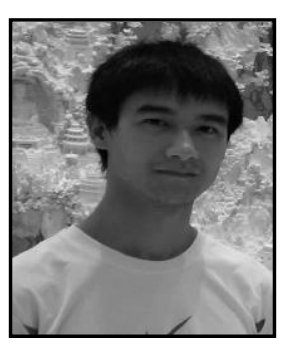

Ping He was born in Nanchong, Sichuan, People's Republic of China, in October 1990. He received the Bachelor degree of Engineering from the School of Automation and Electronic Information, Sichuan University of Science \& Engineering at Zigong, Sichuan, China, in June 2012. He received the Master degree of Engineering from the School of Information Science \& Engineering, Northeastern University at Shenyang, Liaoning, China, in July 2014. He is currently a PhD degree candidate and Research Assistant in the Department of Electromechanical Engineering, Faculty of Science and Technology, University of Macau, Taipa, 999078, Macao Special Administrative Region of China, from September 2014. His main research interests include Control of PDEs, Synchronization of complex networks, Consensus of multi-agent systems, Nonlinear systems.

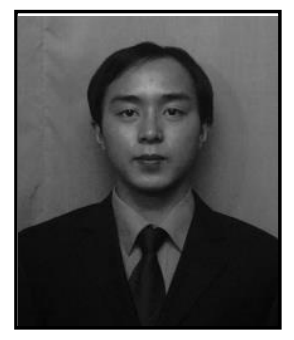

Tao Fan received the B.S. Degree and the M.S. Degree in Measurement Technology and Automation Device from Beijing University of Chemical Technology, in Beijing, People's Republic of China, in 2003 and 2006, respectively. He is currently a Lecturer with the School of Automation and Electronic Information, Sichuan University of Science \& Engineering, in Zigong, Sichuan, China. His main research interests include Intelligent control, Chaos control and synchronization, Complex networks and Consensus of multi-agent systems.

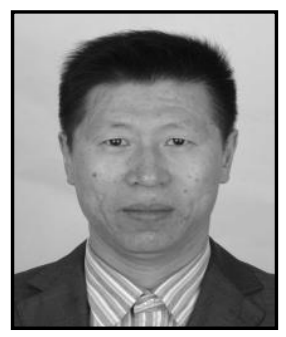

Chun-Guo Jing received the B.S. degree in Information Engineering from Chengdu University of Science and Technology (where is now in Sichuan University), in Chengdu, China and the M.S. degree in Computer Science and Technology from Northeastern University in Shenyang, Liaoning, China, and the $\mathrm{Ph} . \mathrm{D}$. degree in Detection Technology and Automatic Equipment from Yanshan University, in Qinhuangdao, China, in 1990, 2002 and 2008, respectively. $\mathrm{He}$ is currently an Associate Professor with the School of Computer and Communication Engineering, Northeastern University at Qinhuangdao. His main research interests include Multiphase measurement and Application of wireless sensor networks. 